\title{
RELACIÓN ENTRE EL TIEMPO DE USO DE LAS REDES SOCIALES EN INTERNET $Y$ LA SALUD MENTAL EN ADOLESCENTES COLOMBIANOS
}

\author{
ADRIANA PAOLA RODRÍGUEZ PUENTES* \\ ANTONIO FERNÁNDEZ PARRA** \\ UNIVERSIDAD DE GRANADA - ESPAÑA
}

Recibido, diciembre 10/2013

Concepto evaluación, diciembre 20/2013

Aceptado, diciembre 28/2013
Referencia: Rodríguez Puentes, A.P. \& FernándezParra, A. (2014). Relación entre el tiempo de uso de las redes sociales en internet y la salud mental en adolescentes colombianos. Acta Colombiana de Psicología, 17(1), 131-140. doi: 10.14718/ACP.2014.17.1.13

Resumen

\begin{abstract}
El objetivo de esta investigación es determinar si hay diferencias entre grupos de adolescentes de grado $7^{\circ}$ de la ciudad de Bogotá, con diferentes tiempos de uso de las redes sociales en problemáticas de tipo internalizante, externalizante, la soledad percibida, el funcionamiento familiar y el sexo. El estudio con diseño ex post facto valoró el efecto del tiempo de uso de redes sociales a tres niveles (bajo, medio y alto) a través de un cuestionario socio demográfico diseñado para tal fin: el LSRQ, el FACES III, CDI y el YSR. Participaron 96 estudiantes con un rango de edades entre los 11 y 15 años. Se encontró que entre los participantes con un elevado tiempo de uso de las redes sociales, eran mayores los problemas de tipo externalizante (conducta agresiva, ruptura de reglas y problemas de atención). Se discute la necesidad de que en futuras investigaciones se enfaticen los factores positivos que para el desarrollo de los adolescentes puede tener el uso de las redes sociales a través de Internet. Palabras clave: Internet, redes sociales, adolescentes, conductas internalizantes, conductas externalizantes, cualidades positivas
\end{abstract}

\section{RELATIONSHIP BETWEEN THE TIME SPENT ON INTERNET SOCIAL NETWORKING AND MENTAL HEALTH IN COLOMBIAN ADOLESCENTS}

\begin{abstract}
The aim of this study was to explore the relationship between the amount of time spent in social networking and the presence of internalizing and externalizing behavior problems in adolescents. Participants were 96 seventh grade students aged between 11 and 15 years who were attending schools in Bogotá, Colombia. An ex-post facto design was used and the amount of time spent in social networking was divided into three categories: high, medium and low. The instruments included a sociodemographic questionnaire especially designed for this purpose, the Loneliness and Social Dissatisfaction Questionnaire by Cassidy \& Asher (LSDQ for its English acronym), the Youth Self- Report by Achenbach (YSR, for its English acronym), the Child Depression Inventory by Kovacs (CDI, for its English acronym) and the Family Adaptability and Cohesion Evaluation Scales by Olson, Portner \& Lavee (FACES III, for its English acronym). Findings showed that participants with a greater amount of time spent on social networking had more externalyzing disorders (aggressive behavior, rule breaking and attention deficits) than students in the other two categories. The need for future research that stresses the positive factors that the use of Internet social networking can have on adolescents' development is discussed.

Key words: Internet, social networking, adolescents, internalizing behavior, externalizing behavior, positive qualities.
\end{abstract}

* arodriguezp@sdis.gov.co - lunaprin@hotmail.com

** Facultad de Psicología, Campus Cartuja. Universidad de Granada. 18071 Granada, España. afparra@ugr.es. 


\title{
RELAÇÃO ENTRE O TEMPO DE USO DAS REDES SOCIAIS NA INTERNET E A SAÚDE MENTAL DOS ADOLESCENTES COLOMBIANOS
}

Resumo

\begin{abstract}
El objetivo desta pesquisa é determinar se existem diferenças entre grupos de adolescentes da $7^{\circ}$ série da cidade de Bogotá, com diferentes tempos de uso das redes sociais em problemáticas de tipo internalizante, externalizante, a solidão percebida, o funcionamento familiar e o sexo. O estudo com desenho ex pós fato avaliou o efeito do tempo de uso de redes sociais em três níveis (baixo, médio e alto) através de um questionário sóciodemográfico desenhado para esse fim: o LSRQ, o FACES III, CDI e o YSR. Participaram 96 estudantes da faixa etária de 11 a 15 anos. Encontrou-se que entre os participantes com um elevado tempo de uso das redes sociais, eram maiores os problemas de tipo externalizante (conduta agressiva, ruptura de regras e problemas de atenção). Discute-se a necessidade de que em pesquisas futuras sejam enfatizados os fatores positivos que para o desenvolvimento dos adolescentes pode ter o uso das redes sociais através de Internet.

Palavras chave: Internet, redes sociais, adolescentes, condutas internalizantes, condutas externalizantes, qualidades positivas
\end{abstract}

La adolescencia es una etapa de transición entre la niñez y la adultez con cambios madurativos de orden fisiológico, cognitivo, afectivo y psicosocial, factores que la predisponen al desarrollo de problemáticas de carácter psicopatológico. El 20\% de los adolescentes de todo el mundo tiene problemas mentales o de comportamiento (Unicef, 2011) y la mitad de los trastornos mentales comienza antes de los 14 años de edad. La frecuencia de sintomatología depresiva en adolescentes en Colombia es alta, afectando a un 27,5\%; además, el 7,7\% del total de los adolescentes estudiados ha presentado algún trastorno de ansiedad (Torres Galvis, 2010).

Según Judith (2002), a lo largo de esta etapa disminuye la cohesión familiar percibida por parte de los adolescentes, lo cual es un aspecto esperable de esta etapa de desarrollo que se asocia negativamente con problemas externalizantes de comportamiento (Melissa \& Clare, 2006) y con depresión (Murdock, 2011).

Al hablar de salud mental es importante conocer cómo la incorporación de Internet y las nuevas tecnologías en las últimas décadas, no sólo ha provocado un cambio sustancial en la forma de entender el mundo, sino que ha introducido nuevas pautas de relación para el adolescente, tanto con su grupo de pares, como con su familia y con ellos mismos. Todo esto constituye una circunstancia tan significativa, que se identifica a esta generación con el apellido de generación digital, generación interactiva (Del Río, Sádaba \& Bringué, 2010)o la Generación@ (Rubio Gil, 2009).

Según Luengo (2004), las redes sociales en Internet son comunidades virtuales que funcionan como una plataforma de comunicaciones y formas de interacción social, definidas como un intercambio dinámico entre personas, grupos e instituciones. El acceso a ellas se da a través del Internet, denominado red de redes, que involucra la virtualidad. Esta modalidad supera las barreras espaciotemporales, por lo que se hace necesario reconocer los cambios que se dan en el desarrollo social y cognoscitivo.
Por esta razón, sobre Internet y las redes sociales se han formulado planteamientos muy controvertidos, que van desde la asociación de su uso con algunos problemas de salud mental, hasta la consideración de la red como potenciadora de habilidades y facilitadora de la promoción de relaciones sociales y familiares.

La evolución tecnológica plantea un periodo de adaptación a las nuevas formas de adquirir información y comunicación, que puede generar trastornos o desadaptaciones psicológicas más o menos permanentes. Una de las posturas más desarrolladas en este sentido es la que considera el uso de Internet como adicción (Echeburúa, Labrador \& Becoña, 2009). Dentro de ella hay estudios, como el realizado por Morrison \& Gore (2010), quienes encontraron que los adolescentes que se consideran a sí mismos dependientes del Internet presentan niveles más altos de depresión. Otros estudios han relacionado el uso de Internet con problemas de tipo externalizante, como el comportamiento agresivo (Ko, Yen, Liu, Huang \& Yen, 2009). También Cao, Su, Wan, Hao \& Tao (2011) encontraron que los adolescentes chinos usuarios de Internet presentan mayor probabilidad de sufrir síntomas psicosomáticos y problemas de adaptación social que los no usuarios.

Respecto a las posturas positivas, Borzekowski (2006) ha enfatizado lo productivo que puede resultar Internet en función del uso que se le dé. Así, Tabernero, Aranda \& Sánchez-Navarro (2010) encontraron que en España el uso principal de las redes sociales por parte de los adolescentes es hablar con amigos (78\%). Según Albero (2002), Internet amplía los formas de socialización de los adolescentes, quienes lo integran en su cotidianidad a partir de parámetros culturales, y su uso contribuye a marcar sus intereses como grupo.

El estudio realizado por Sánchez \& Martin (2011) en Argentina, Colombia, Ecuador, España, México y Venezuela, halló que entre el $80 \%$ y el $90 \%$ de los adolescentes en edad 
escolar emplean las redes sociales, siendo los adolescentes colombianos los que mostraban el porcentaje más alto de uso. Según el propio Ministerio de Tecnologías de la Información y las Comunicaciones (2010), en Colombia el $52 \%$ de la población utiliza diariamente Internet por un promedio de tres horas, presentándose un porcentaje de uso por parte de los adolescentes del $98 \%$.

La investigación realizada por Viñas (2009) encontró que los adolescentes con alto uso del Internet buscan lazos online, debido a la intimidad acelerada que promueve este medio. En la medida que la interacción con las nuevas tecnologías puede relacionarse con esta búsqueda, es relevante considerar la percepción de soledad de los adolescentes, ya que se ha encontrado que en esta etapa la soledad percibida puede estar asociada con estados emocionales como la depresión (Heinrich \& Gullone, 2006).

En la adolescencia, las relaciones con pares tienen mayor valor que en otras etapas de la vida. Los adolescentes intentan desarrollar un mayor grado de autonomía a costa del distanciamiento de sus padres; se encuentra que la ausencia de relaciones significativas con pares está asociada con bajo bienestar psicológico de los adolescentes (Laible, 2007).

En un estudio longitudinal realizado por Fontaine et al. (2009) se halló que la escacez de preferencias sociales en edades tempranas conlleva sentimientos de soledad, lo que puede conducir a problemas de ansiedad y depresión en la adolescencia. Igualmente, la percepción que tiene el adolescente de las relaciones sociales, de si son adecuadas o no, juega un papel más significativo en el desarrollo de problemas que la medida objetiva de redes sociales (Qualter, Braw, Munn \& Rotenberg, 2010).

En una investigación con estudiantes universitarios se encontró que el hecho de pasar más tiempo en Internet interfería en sus relaciones sociales, debido a que tenían que cambiar sus citas con amigos por estar en Internet, y adicionalmente les acarreaba más problemas con su familia (Muñoz-Rivas, Fernández \& Gámez-Guadiz, 2010).

Algunos autores se han centrado en determinar a partir de cuánto tiempo el uso de Internet puede considerarse como problemático. Shapira, Goldsmith, Keck, Khosla \& McElroy (2000) encontraron que después de unas 20 a 28 horas semanales, su uso empieza a ocasionar dificultades. En el estudio de Forero, Sala, \& Chalezquer (2010) se considera que el $45,7 \%$ de los adolescentes son "usuarios fuertes", aunque en ningún caso este uso sustituye la comunicación personalizada. Sánchez \& Martin (2011) encontraron que los adolescentes emplean principalmente las redes sociales para mantener el contacto con aquellas personas que ven regularmente, pero también para mantener la relación con otras personas a las que no ven de manera habitual. Por su parte, Kaveri \& Lin (2007) encontraron que el tiempo de uso de Internet no está relacionado ni con la soledad, ni con el soporte social percibido, pero sí con el apoyo de sus padres, ya que quienes reportaron menos apoyo parental tendían a buscar más relaciones online.

La dedicación a las tecnologías digitales está transformando el aprendizaje, la socialización y la comunicación entre los jóvenes que pueden acceder a ellas y usarlas, generando nuevas formas de expresión asociadas, que conllevan nuevas dinámicas relacionales.

De acuerdo con la revisión anterior, es evidente que no hay suficiente investigación en Colombia respecto al uso de internet y de las redes sociales, ni de su relación con la salud mental, la soledad percibida, el clima familiar y el nivel socioeconómico de los adolescentes. Por lo tanto, se planteó este trabajo para analizar estas variables en adolescentes de séptimo grado procedentes de colegios públicos y privados de la ciudad de Bogotá. Con este estudio se pretendía conocer si existen diferencias entre adolescentes en función del tiempo dedicado a las redes sociales en internet, respecto a: a) sus problemas psicológicos de tipo internalizante y externalizante; b) la soledad percibida por los participantes; c) los niveles de funcionamiento familiar; d) tener acceso a Internet en los hogares, y e) el sexo. El Estudio tiene un diseño ex post facto (experimento natural o estudio cuasiexperimental) con una única variable independiente (tiempo en internet) no manipulada, sino seleccionada de forma natural (Montero \& León, 2007). En este caso se seleccionaron los participantes por poseer un determinado valor en el tiempo de uso de internet.

\section{MÉTODO}

\section{Participantes}

Participaron en este estudio 96 adolescentes escolarizados en $7^{\circ}$ grado, 43 hombres y 53 mujeres, con edades comprendidas entre los 11 y 15 años de edad $(M=11.98$; $D T=0,68)$. De ellos, 24 participantes $(25 \%)$ procedían de un colegio público-concertado ubicado en la localidad de Bosa; 47 (49\%), de un colegio privado ubicado en la localidad de Rafael Uribe Uribe, y 25 (26\%), de un colegio privado en la localidad de Fontibón. Todos se encontraban en jornada escolar única. Un total de 51 adolescentes (53\%) pertenecían al estrato socioeconómico medio bajo; 27 (28\%), al bajo; doce (13\%), al medio; cuatro (4\%), al medio alto, y dos (2\%), al muy bajo.

Las instituciones escolares facilitaron el acceso, por lo que el muestreo fue de tipo no probabilístico por conveniencia. Para la formación de los tres grupos de estudio, los participantes se dividieron de acuerdo con el tiempo de uso diario de las redes sociales en Internet. El primer grupo (Bajo Uso) estuvo compuesto por aquellos participantes que 
pasaban menos de una hora al día utilizando las redes sociales. El segundo grupo (Uso Medio) lo componían quienes hacían uso de las redes sociales de una a dos horas por día, y el tercer grupo (Alto Uso), de tres horas en adelante. El grupo de Bajo Uso estuvo compuesto por 29 participantes, el grupo de Uso Medio por 42, y el de Alto Uso por 25.

\section{Instrumentos}

Cuestionario de Insatisfacción Socialy Soledad. (LSDQ - Loneliness and Social Dissatisfaction Questionnaire) de Cassidy \& Asher (1992). Este autoinforme contiene un total de 24 ítems que evalúan hasta qué punto los niños se sienten solos y socialmente insatisfechos en el contexto escolar; de éstos, 16 ítems miden el sentimiento de soledad y la insatisfacción social en el contexto escolar. Los ocho ítems restantes son suplementarios; es decir, hacen referencia a aspectos de la vida diaria y ocio del informante. La versión utilizada fue adaptada al español por Spridou \& Fernández Parra (2008) siguiendo las directrices de Muiñiz \& Hambleton (2000). Los autores informan de una consistencia interna satisfactoria de 0,79 . No existe una versión adaptada específicamente para población colombiana; sin embargo, se comprobó a través de juicio de expertos, que la versión española utilizada no tenía problemas de comprensión.

Autoinforme del Comportamiento de Jóvenes de 11-18 años. (YSR - Youth Self-Report) de Achenbach (1991). Es un autoinforme de screening de conductas psicopatológicas en adolescentes. La estructura original del cuestionario contempla una escala de competencias y otra de problemas de conducta que consta de 112 ítems, de los cuales 96 hacen referencia a conductas problemáticas y 16 a conductas prosociales. El rango de puntuación va de 0 a 2 (donde 0 = frase no cierta, $1=$ frase algo cierta o te sucede a veces, $2=$ lo que dice es muy cierto o te ocurre frecuentemente). Se ha informado de valores de alfa de Cronbach de 0,61 y 0,67 para chicos y chicas, respectivamente. En este estudio se utilizó la versión internacional en español de 2001.

Inventario de Depresión Infantil. (CDI - Child Depressive Inventory) de Kovacs (2004). Se trata de una escala autoaplicable para edades comprendidas entre los siete y los 17 años. Está formada por 27 ítems con tres alternativas de respuesta, puntuables de 0 a 2 en función de la ausencia, la menor o mayor gravedad de síntoma reflejado en la respuesta. La puntuación total del sujeto se obtiene sumando todos los reactivos, y puede oscilar entre 0 y 54 puntos; cuanto mayor sea la puntuación obtenida, mayor es la patología depresiva. La prueba ha demostrado su fiabilidad, con un alfa de Cronbach para la escala total de 0,80 , una consistencia interna de 0,82 en población española (Del
Barrio, Moreno \& López, 1999, 2001, citados en Kovacs, 2004). En un estudio sobre la validación y las características psicométricas de esta prueba en niños colombianos, se determinó un alfa de Cronbach de 0.84 , lo cual indica que los reactivos sí están diseñados para medir síntomas de depresión infantil en población colombiana (Segura, Posada, Ospina, M., \& Ospina, 2010). En este estudio se utilizó la versión oficial en español, la cual no presenta dificultades de comprensión para adolescentes colombianos, según el juicio de expertos realizado.

Escala de Evaluación de Adaptabilidad y Cohesión Familiar III. (FACES III - The Family Adaptability and Cohesion Evaluation Scales) de Olson, Portner \& Lavee (1985), y traducida al español por Ponce-Rosas, GómezClavelina, Terán Trillo, Irigoyen-Coria \& LandgraveIbáñez (2002). Presenta un adecuado nivel de fiabilidad en ambas dimensiones teóricas, 0,89 en Cohesión y 0,87 en Adaptabilidad. Es un instrumento de autoinforme que evalúa dos funciones principales a través de 20 reactivos (diez para adaptabilidad y diez para cohesión). Se aplica a personas mayores de diez años que sean miembros de familias con hijos y que sepan leer y escribir. Cuenta con una escala que incluye cinco opciones (nunca, casi nunca, algunas veces, casi siempre y siempre) con valores de 1 a 5. Se debe contestar el cuestionario en cuanto a la forma en que el entrevistado considera que su familia reaccionaría habitualmente.

Cuestionario de Variables Socio Demográficas. Diseñado para esta investigación, incluía preguntas que se consideraron pertinentes para los objetivos propuestos: bienes que tienen en sus hogares, el acceso a Internet, horas que permanecen solos en casa entre y fines de semana, o tiempo que pasan en Internet usando las redes sociales, así como datos sobre la escolaridad, edad de los participantes, sexo y caracterización social.

\section{Procedimiento}

Se contactó a los centros educativos del Distrito Capital de Bogotá a través de las orientadoras escolares. En el primer encuentro se explicó el objetivo de la investigación, obteniendo el permiso para la aplicación de los instrumentos, y se acordó la fecha de entrega de los consentimientos informados. En la segunda visita se hizo entrega de dicho documento para que los estudiantes lo llevaran a su casa y se lo proporcionaran a sus padres. El documento de consentimiento informado se acompañó de una circular explicativa y de los datos de contacto.

Se realizó la aplicación de los instrumentos de evaluación de manera grupal. Se indicó a los participantes que la 
Tabla 1

Comparaciones entre grupos respecto a variables sociodemográficas y uso de Internet.

\begin{tabular}{|c|c|c|c|c|c|}
\hline Grupos & Alto Uso $(n=29)$ & Uso Medio $(n=42)$ & Bajo Uso $(n=25)$ & $\mathrm{Chi}^{2}$ & $p$ \\
\hline \multicolumn{6}{|l|}{ Sexo } \\
\hline Hombre & 7 & 22 & 14 & 7,251 & $0,027^{*}$ \\
\hline Mujer & 22 & 20 & 11 & & \\
\hline \multicolumn{6}{|l|}{ Convivencia } \\
\hline Ambos progenitores & 20 & 31 & 20 & 3.805 & 0.433 \\
\hline Un progenitor & 7 & 9 & 1 & & \\
\hline Otras personas & 2 & 2 & 4 & & \\
\hline \multicolumn{6}{|l|}{ Acceso a Internet } \\
\hline Sí & 28 & 39 & 15 & 15,794 & $0,001^{*}$ \\
\hline No & 1 & 3 & 10 & & \\
\hline
\end{tabular}

$* p<0,05$

evaluación no tendría implicaciones en el ámbito académico, se explicó el objetivo de la investigación y resolvieron las dudas planteadas. Los estudiantes respondieron los instrumentos de manera anónima y se les asignó un código. El tiempo máximo de aplicación fue de una hora y 45 minutos para los primeros instrumentos, y de una hora y 20 minutos en la segunda aplicación, con recesos para el descanso de los participantes.

\section{RESULTADOS}

Respecto a las variables de tipo demográfico y social, se encontraron diferencias significativas en la distribución de ambos sexos entre los grupos, tal y como muestra la tabla 1. Los datos indican que mientras en el grupo de Alto Uso de las redes sociales había más mujeres que hombres, ocurría lo opuesto en el grupo de Bajo Uso. De los 96 adolescentes, 71 viven con ambos progenitores, 17 con uno de ellos, y 8 con otras personas, sin que se encontraran diferencias significativas entre grupos. Sí hubo diferencias, sin embargo, respecto al acceso a Internet, ya que en los grupos de Alto y Medio Uso de redes sociales, sólo cuatro participantes no tenían Internet en casa, mientras que la cifra se elevaba a diez en el grupo de Bajo Uso. No se encontraron diferencias significativas entre los grupos, en relación con la distribución por estrato social.

Tabla 2

Comparaciones entre grupos respecto a los problemas psicológicos reportados por los adolescentes a través del YSR de Achenbach (1991).

\begin{tabular}{lcccccccc}
\hline \multicolumn{1}{c}{ Grupos } & \multicolumn{2}{c}{ Alto Uso $(\boldsymbol{n}=\mathbf{2 5})$} & \multicolumn{2}{c}{ Uso Medio $(\boldsymbol{n}=\mathbf{4 2})$} & \multicolumn{2}{c}{ Bajo Uso $(\boldsymbol{n}=\mathbf{2 9})$} & & P \\
\hline \multicolumn{1}{c}{ Escalas } & Media & DT & Media & DT & Media & DT & $\boldsymbol{F}$ & P \\
\hline Ansiedad/ Depresión & 7,83 & 3,742 & 7,21 & 3,679 & 5,88 & 3,140 & 1,162 & 0,318 \\
Aislamiento/ Depresión & 4,28 & 2,186 & 5,24 & 2,593 & 4,16 & 2,322 & 2,017 & 0,139 \\
Quejas Somáticas & 5,48 & 2,886 & 5,17 & 2,613 & 5,16 & 4,007 & 0,012 & 0,988 \\
Problemas Sociales & 5,41 & 3,224 & 5,62 & 2,930 & 4,68 & 2,839 & 0,863 & 0,425 \\
Problemas Pensamiento & 4,86 & 3,701 & 3,83 & 2,767 & 3,20 & 2,550 & 2,343 & 0,102 \\
Problemas Atención & 7,62 & 3,886 & 6,17 & 3,177 & 5,16 & 2,357 & 3,768 & $0,027^{*}$ \\
Ruptura Reglas & 7,59 & 3,850 & 6,14 & 3,640 & 5,24 & 2,862 & 3,368 & $0,039^{*}$ \\
Conducta Agresiva & 12,17 & 6,376 & 9,71 & 4,676 & 8,48 & 4,389 & 3,840 & $0,025^{*}$ \\
Problemas Internalizantes & 17,59 & 6,722 & 17,62 & 6,886 & 15,20 & 7,676 & 0,714 & 0,492 \\
Problemas Externalizantes & 19,76 & 9,665 & 15,86 & 7,436 & 13,72 & 6,341 & 4,342 & $0,016^{*}$ \\
Cualidades Positivas & 19,93 & 2.915 & 17,83 & 3,957 & 16,68 & 4,100 & 3,543 & $0,033^{*}$ \\
\hline Total YSR & 60,83 & 23,896 & 54,26 & 20,022 & 46,28 & 17,341 & 2,831 & 0,064 \\
\hline
\end{tabular}

$* \mathrm{p}<0.05$ 
Otras variables analizadas fueron el rendimiento escolar medio, que fue de $1,96(D T=0,49)$ en una escala entre 0 y 3 , para la totalidad de la muestra. No se encontraron diferencias significativas entre los grupos $(F=0,033, p=$ $0,967)$, ya que el rendimiento medio del grupo de Alto Uso fue de 1,96 ( $D T=0,53)$, el de Uso Medio fue de 1,94 (DT $=0,43)$ y el de Uso Bajo fue de $1,91(D T=0,56)$.

Puesto que se encontraron diferencias entre los grupos en la distribución de participantes de ambos sexos, se procedió a realizar una ANOVA 3 (niveles de uso) x 2 (sexo). Respecto al efecto principal de los niveles de uso, la Tabla 2 recoge los resultados obtenidos en cada uno de los grupos sobre los problemas o escalas principales evaluadas a través del YSR. Se realizaron entonces comparaciones post hoc en aquellas variables en las que hubo diferencias significativas entre los tres grupos. Se encontraron diferencias significativas entre los grupos de Alto Uso y Bajo Uso en la escala de banda ancha de problemas de tipo externalizante (Dif. de Medias $=6,04, p=0,020$ ), la escala de conducta agresiva (Dif. de Medias $=3,69, p=0,035)$, la de ruptura de reglas (Dif. de Medias $=2,35, p=0,045)$ y la de problemas atencionales (Dif. de Medias $=2,46, p=0,020$ ), así como en la variable cualidades positivas (Dif. de Medias $=3,25, p=0,005$ ). En todos los casos la puntuación obtenida por el grupo de Alto Uso de las redes sociales fue superior a la obtenida por el grupo de Bajo Uso.
En la tabla 3 se presentan los resultados obtenidos en cada uno de los grupos en relación con los problemas psicológicos evaluados a través del CDI, LSDQ, y la funcionalidad familiar medida a través del FACES III. No se encontraron diferencias significativas entre los grupos.

Finalmente, se valoraron las diferencias en función de la variable Sexo, sin que se hallaran diferencias significativas en función de esta variable para la mayoría de las variables estudiadas, salvo los problemas somáticos valorados en el YSR, así como en la escala de problemas internalizantes y problemas de conducta. Los resultados se muestran en la tabla 4. En todos los casos la puntuación media del grupo de mujeres fue significativamente superior a la puntuación obtenida por los hombres, salvo en la escala de problemas de conducta. No hubo efectos de interacción entre las variables tipo de uso y sexo.

Por último, se realizó un análisis de regresión múltiple con todas las variables que podrían guardar una relación de tipo predictivo. Al finalizar el análisis se encontró que la conducta agresiva, las horas a la semana que permanecen solos, las cualidades y la insatisfacción escolar, predicen el tiempo de uso diario de las redes sociales en Internet, siendo la conducta agresiva la que guarda mayor poder predictivo respecto al tiempo que pasan los adolescentes en Internet en las redes sociales. En la tabla 5 se recogen dichos resultados.

Tabla 3

Comparación entre grupos respecto a las escalas del LSDQ, FACES III y CDI

\begin{tabular}{|c|c|c|c|c|c|c|c|c|}
\hline \multirow{2}{*}{$\begin{array}{l}\text { Grupos } \\
\text { Escalas }\end{array}$} & \multicolumn{2}{|c|}{ Alto Uso $(n=25)$} & \multicolumn{2}{|c|}{ Uso Medio $(n=42)$} & \multicolumn{2}{|c|}{ Bajo Uso $(n=29)$} & \multirow[b]{2}{*}{$F$} & \multirow[b]{2}{*}{$\mathbf{P}$} \\
\hline & Media & DT & Media & DT & Media & DT & & \\
\hline \multicolumn{9}{|l|}{ LSDQ $^{1}$} \\
\hline Insatisfacción Escolar & 20,51 & 3,685 & 20,29 & 3,172 & 19,84 & 4,346 & 0,282 & 0,755 \\
\hline Soledad & 1,17 & 1,197 & 0.48 & .0552 & 0.48 & 0.510 & 0,140 & 0,870 \\
\hline Total & 22,59 & 3,397 & 21,40 & 2,669 & 21,16 & 3,520 & 0,273 & 0,762 \\
\hline \multicolumn{9}{|l|}{$\mathrm{CDI}^{2}$} \\
\hline Disforia & 4,72 & 3,654 & 4,57 & 3,736 & 4,28 & 3,446 & 0,184 & 0,833 \\
\hline Autoestima & 6,93 & 2,434 & 6,64 & 3,138 & 5,92 & 2,482 & 1,394 & 0,253 \\
\hline Total & 11,66 & 5,115 & 11,21 & 6,159 & 10,29 & 4,813 & 0,727 & 0,486 \\
\hline \multicolumn{9}{|l|}{ FACES III $^{3}$} \\
\hline Cohesión & 35,62 & 5,894 & 36,95 & 4,813 & 35,68 & 7,151 & 0,385 & 0,682 \\
\hline Adaptabilidad & 28,21 & 5,130 & 29,90 & 5,481 & 26,72 & 4,739 & 2,685 & 0,074 \\
\hline Total & 62,83 & 8,706 & 66,86 & 8,839 & 62,40 & 10,21 & 1,768 & 0,176 \\
\hline
\end{tabular}


Tabla 4

Diferencias entre hombres y mujeres en variables del estudiol.

\begin{tabular}{|c|c|c|c|c|c|c|}
\hline Grupos & Ho & & & & & \\
\hline Escalas & Media & DT & Media & DT & F & $\mathrm{P}$ \\
\hline Somáticos & 4,40 & 2,838 & 5,96 & 3,113 & 5,733 & $0,019 *$ \\
\hline Internalizantes & 15,26 & 6,644 & 18,38 & 7,131 & 5,056 & $0,027 *$ \\
\hline Problemas Conducta & 6,37 & 4,364 & 4,74 & 3,415 & 4,865 & $0,030 *$ \\
\hline
\end{tabular}

${ }^{1}$ Sólo se presentan las variables en las que se encontraron diferencias significativas entre ambos sexos, todas ellas del cuestionario YSR de Achenbach (1991).

$* p<0.05$

$\underline{\text { Tabla } 5}$

Análisis de Regresión lineal múltiple: variables predictoras del tiempo de uso de las redes sociales en Internet

\begin{tabular}{|c|c|c|c|c|c|c|}
\hline & & Beta & Media Cuadrática & $R^{2}$ & $F$ & $p$ \\
\hline Modelo 1 & Conducta agresiva & 0,367 & 37,033 & 0,134 & 14,590 & $0,001 *$ \\
\hline \multirow[t]{2}{*}{ Modelo 2} & Conducta agresiva & 0,329 & 27,188 & 0,197 & 7,290 & $0,008^{*}$ \\
\hline & Horas solo a la semana & 0,254 & & & & \\
\hline \multirow[t]{3}{*}{ Modelo 3} & Conducta agresiva & 0,289 & 23,015 & 0,251 & 6,533 & $0,012 *$ \\
\hline & Horas solo a la semana & 0,250 & & & & \\
\hline & Cualidades Positivas & 0,234 & & & & \\
\hline \multirow[t]{4}{*}{ Modelo 4} & Conducta agresiva & 0,357 & 19,962 & 0,290 & 5,022 & $0,027 *$ \\
\hline & Horas solo a la semana & 0,222 & & & & \\
\hline & Cualidades Positivas & 0,218 & & & & \\
\hline & Insatisfacción escolar & 0,210 & & & & \\
\hline
\end{tabular}

$* \mathrm{p}<0.05$

\section{DISCUSIÓN}

Considerando la información aportada por los propios adolescentes a través de instrumentos de autoinforme, se hallaron diferencias significativas entre los tres grupos de participantes, divididos según el tiempo de uso de las redes sociales, respecto a diferentes problemas psicológicos. Así, los adolescentes con un Alto uso de redes sociales, aquellos que empleaban más de tres horas diarias en Internet, mostraban un nivel mayor de problemas externalizantes evaluados mediante el YSR, tanto en la escala global o de banda ancha, como en las escalas de conducta agresiva, ruptura de reglas y problemas de atención. Ese mayor nivel de problemas externalizantes se puede considerar coherente con otros estudios en los que se ha encontrado que los adolescentes que presentaron comportamiento agresivo tenían más probabilidades de usar Internet cada día, y más de 20 horas por semana (Ko et al., 2009). El uso de Internet se ha visto también asociado al trastorno por déficit de atención e hiperactividad (González \& De la
Rosa, 2007). Sin embargo, otros estudios muestran el uso de Internet como potenciador del área cognitiva, ya que lo consideran como un complejo gigantesco de red virtual de redes, que permite mejorar el desarrollo cognitivo y de este modo contribuir a procesos atencionales, puesto que las personas en la red desarrollan procesos de atención dividida o simultánea (Greenfield y Yan, 2006).

Como parte de los aspectos positivos en esta investigación, se han analizado las diferencias entre los grupos respecto a las cualidades positivas informadas por los participantes a través del YSR. Se encontró que los adolescentes que pasan más tiempo en las redes sociales en Internet (Alto Uso), mostraban un mayor nivel de cualidades. De acuerdo con Castellana, Sánchez, Graner \& Beranuy (2007), entre los efectos positivos del uso de Internet figura que facilita la interacción. Además, los propios adolescentes perciben que su relación con las redes sociales es muy saludable (Sánchez \& Martin, 2011).

En contravía a lo que plantea la literatura, no se han encontrado diferencias significativas entre grupos de 
participantes respecto a problemas de tipo internalizante, a pesar de que los propios adolescentes informaron sobre ellos. No se diferencia entre grupos de uso de las redes sociales respecto a variables como la soledad percibida, la insatisfacción social escolar, disforia y autoestima. Esto es coherente con lo descrito en la literatura que plantea que los adolescentes usan las redes sociales con fines de comunicación y para ampliar las formas de socialización. De esta forma, los adolescentes incorporan las redes sociales a través de Internet a su diario vivir, con lo que afianzan sus intereses como grupo (Albero, 2002; Castellana et al., 2007).

Tampoco se encontraron diferencias entre los grupos con distinto nivel de uso de las redes sociales respecto al funcionamiento familiar, valorado mediante el cuestionario FACES III. Este resultado contrasta con ciertos estudios que indican que el uso de Internet genera una afectación positiva o negativa en las relaciones familiares, esta última con el argumento de que aumenta la brecha generacional, además del peligro percibido por los progenitores en el uso de estos medios (Mesch, 2003). Sin embargo, hay estudios que encuentran efectos positivos, atribuíbles a que los adolescentes pueden permanecer más tiempo en casa, lo cual conlleva un incremento de la comunicación con sus padres, generando nuevas formas de interacción (Barrera, 2012). En todo caso, en este estudio el funcionamiento familiar fue valorado por los propios adolescentes.

Se han encontrado cuatro variables con valor predictivo del tiempo de uso de Internet: la conducta agresiva, el tiempo que los adolescentes permanecen solos en casa, las cualidades de los adolescentes y, por último, la insatisfacción social de los menores. Respecto a la conducta agresiva, debe resaltarse que Ko et al. (2009) encontraron que los adolescentes con adicción a Internet presentaban en el año previo a la evaluación más comportamientos de tipo agresivo. Respecto al tiempo que permanecen solos, aunque no hay estudios que indaguen en dicha relación, puede ser que los progenitores o cuidadores, al estar presentes, ejercen algún tipo de control sobre el tiempo de uso de Internet, y en este caso, de las redes sociales. En relación con las cualidades positivas, no analizadas en estudios previos, es difícil valorarlas al tratarse de una variable de tipo positivo. Finalmente, la insatisfacción escolar, evaluada mediante el LSDQ, está vinculada con la insatisfacción en las relaciones con pares en el ámbito escolar. Es posible que la relación encontrada se deba a que los adolescentes se sienten inconformes con sus relaciones en el ámbito escolar, lo que les lleva a emplear más tiempo a diario relacionándose a través de las redes sociales, para ser honestos en aspectos que no discutirían fácilmente frente a frente (Castellana et al., 2007).
En el presente estudio se encontró también que el tiempo de uso de las redes sociales de los adolescentes estaba relacionado con el acceso a Internet desde sus hogares, lo que resulta coherente con otras investigaciones que también han encontrado que el mayor porcentaje de adolescentes se conecta con redes desde su casa (Castellana et al., 2007; Viñas, 2009), siendo también el hogar el lugar desde el que se conectan por más tiempo (Observatorio Nacional de Telecomunicaciones y de la SI, 2011).

Una de las variables de análisis en este estudio fue el sexo. En en cuanto a las diferencias significativas encontradas entre hombres y mujeres respecto al uso de redes sociales a través de Internet, coinciden con otros estudios donde las chicas son más activas en el uso de estas herramientas (Tabernero et al., 2010). Así, la mujer colombiana pasa más tiempo online, aunque no hay datos específicos para este grupo etario (Seguic, 2010). Otros estudios han encontrado diferencias en cuanto al tipo de uso, ya que los adolescentes varones usan el Internet con fines comunicativos, pero difieren de las mujeres en que ellos lo emplean para juegos duros, lo que establece una relación diferente usuario-ordenador (Gross, 2004). En cualquier caso, no se encontró en este estudio una interacción entre el sexo y el tiempo de uso de las redes sociales, y sólo algunas diferencias globales en función del sexo de los participantes. Los niños participantes en este estudio presentan más problemas de conducta externalizada (escala de problemas de conducta del YSR) que las niñas, mientras que estas últimas muestran más problemas somáticos (quejas somáticas) y de tipo internalizante (escala internalizante de banda ancha) en relación con los niños, lo cual ha sido ampliamente validado en la literatura (Caraveo et al., 1992; Valencia \& Andrade-Palos, 2005).

Como limitaciones principales del estudio se destaca el tamaño de la muestra ya que es pequeña, lo cual afecta la generalización de los resultados. También se puede destacar la clase de instrumentos utilizados, todos de autoinforme, por lo que sería pertinente incluir en un futuro estudio a múltiples informantes. De esta manera, sería posible contrastar la información aportada por los propios adolescentes con la que adultos, padres o profesores pueden ofrecer sobre las mismas áreas de comportamiento aquí estudiadas.

Algunos autores han considerado importante la Internet en la vida de los adolescentes, en cuanto potenciador del desarrollo cognoscitivo, como facilitador de la interacción social, por el fenómeno de la desinhibición y la subsecuente asociación que puede darse para los adolescentes (Borzekowski, 2006). Sin embargo, Kraut et al. (1998) ya alertaron sobre lo que llamaron la "paradoja de Internet": aunque pueda ser utilizado para establecer relaciones sociales, puede reducir la implicación social directa de esos 
usuarios y el consiguiente bienestar psicológico. Desde la perspectiva de estos autores, son dos los posibles mecanismos que explicarían esta paradoja. En primer lugar, el tiempo dedicado al uso de Internet, incluso para comunicarse, iría en detrimento de actividades sociales "menos pasivas". En segundo lugar, ese tiempo podría desplazar relaciones sociales directas, que implican vínculos más fuertes con familia y amigos. Desde la perspectiva de estos autores esas relaciones directas serían más satisfactorias y saludables. Por otra parte, un tiempo elevado de uso de las redes sociales de Internet también podría ser entendido como indicativo de abuso o adicción, tal y como han interpretado diversos autores (Echeburúa et al., 2009; Morrison y Gore, 2010). Esta última implicación no ha sido valorada en este estudio.

En conclusión, se puede decir que este estudio ha encontrado que existe una relación entre el tiempo de uso de las redes sociales y ciertas problemáticas autoinformadas de tipo externalizante en adolescentes. Al mismo tiempo, los adolescentes participantes en este estudio que usan las redes sociales a través de Internet se perciben con más cualidades positivas. También se puede destacar que el acceso a Internet se realiza principalmente desde los propios hogares de los adolescentes. A pesar de que las niñas son quienes permanecen más tiempo en las redes sociales, según los datos de este estudio, el sexo no interactúa con el efecto producido por el tiempo de uso de Internet.

Se sugiere para el futuro no limitarse sólo a indagar los efectos negativos del Internet en su vinculación con problemas psicológicos, sino su impacto en las características positivas como la interacción con compañeros, incremento en autoestima, autoeficacia y percepción de habilidades por parte de terceros.

\section{REFERENCIAS}

Achenbach, T. M. (1991). Integrative guide for the 1991 CBCL/4-18, YSR, and TRF profiles. Burlington, VT: University of Vermont, Department of Psychiatry.

Albero, M. (2002). Adolescentes e Internet. Mitos y realidades de la sociedad de la información. Zer: Revista de Estudios de Comunicación, 13, 1-8.

Barrera, V. D. (2012, abril). Usos y significaciones del Internet en familias con hijos adolescentes. III Seminario Nacional de Infancia y Adolescencia, Ciudad de Medellín, Colombia.

Borzekowski, D. (2006). Adolescents use of the Internet: A controversial, coming of-age resource. Adolescent Medicine Clinics, 17, 205-216.

Cao, H., Su, Y., Wan, Y., Hao, J. y Tao, F. (2011). Problematic Internet use in Chinese adolescents and its relation to psychosomatic symptoms and life satisfaction. BMC Public Health , 11, 1-9.

Caraveo, J., Medina, M. E., Tapia, R., Rascón, M., Gómez, M. y Villatoro, J. (1992). Trastornos psiquiátricos en niños de la República Mexicana: Resultados de una encuesta de hogares. Anales del Instituto Mexicano de Psiquiatría, 3, 56-62.

Cassidy, J. y Asher, S. (1992). Loneliness and peer relations in young children. Child Development, 63, 350-365.

Castellana, M., Sánchez, X., Graner, C. y Beranuy, M. (2007). El adolescente ante las tecnologías de la información y la comunicación: Internet, móvil y video juegos. Papeles del Psicólogo, 28, 196-204.

Cuevas, M. y Teva, I. (2006). Evaluación y tratamiento de la depresión infantil. En Bermúdez, M. y Bermúdez, A. (Eds.), Manual de psicología clínica infantil (pp. 219-268). Madrid: Biblioteca Nueva.

Del Rio, J., Sábada, C., Bringué, X. (2010). Menores y redes ¿sociales?: de la amistad al ciberbullying. Revista de Estudios de la Juventud, 88, 115-129.

Echeburúa, E., Labrador, F. J. y Becoña, E. (2009). Adicción a las nuevas tecnologías en adolescentes y jóvenes. Madrid: Pirámide.

Fontaine, R. G., Pettit, G. S., Yang, C., Dodge, K., Burks, V. S., Price, J. M. y Bates, J. E. (2009). Loneliness as a partial mediator of the relation between low social preference in childhood and anxious/depressed symptoms in adolescents. Development and Psychopathology, 21, 479-491.

Forero, G. A., Sala, X. B. y Chalezquer, Ch. S. (2010). La generación interactiva en Colombia: adolescentes frente a la Internet, el celular y los videojuegos. Anagramas, 9, 45-56.

Greenfield, P. y Yan, Z. (2006) Children, adolescents, and the Internet: a new field of inquiry. Developmental Psychology, 42(3), 391-394.

González-Rodríguez, M. P. y De la Rosa, M. V. (2007). La adicción a Internet en adolescentes, se asocia con síntomas psiquiátricos como TDAH, depresión y hostilidad. Evidencias en Pediatría, 3, 104-108.

Gross, E. F. (2004). Adolescent Internet use: What we expect, what teens report. Applied Developmental Psychology, 25, 633-649.

Heinrich, L.M. y Gullone, E. (2006). The clinical significance of loneliness: A literature review. Clinical Psychology Review, 26, 695-718.

Judith, B. (2002). Is family cohesion a risk or protective factor during adolescent development? Journal of Marriage and Family, 64, $668-675$.

Kaveri, S. y Lin, G. (2007). Adolescents on the net: Internet use and well-being. Adolescence 42, 659-677.

Ko, C.H., Yen, J.Y., Liu, S.C., Huang, C.F. y Yen, C.F. (2009). The associations between aggressive behaviors and Internet addiction and online activities in adolescents. Journal of Adolescent Health, 44, 598-605. 
Kovacs, M. (2004). Inventario de depresión infantil (CDI). Madrid: TEA Ediciones. Kraut, R., Patterson, M., Lundmark, V., Kiesler, S., Mukophadhyay, T. y Scherlis, W. (1998). Internet paradox. A social technology that reduces social involvement and psychological well-being? American Psychologist, 53, 1017-1031.

Laible, D. (2007). Attachment with parents and peers in late adolescence: Links with emotional competence and social behavior. Personality and Individual Differences, 43, $1185-1197$.

Luengo, A (2004). Adicción a Internet: conceptualización y propuesta de intervención. Revista Profesional Española de Terapia Cognitivo-Conductual, 2, 22- 52.

Melissa, K. R. y Clare, M. S. (2006). Association between family cohesion and adolescent siblings externalizing behavior. Journal of Family Psychology. 20, 663-669.

Mesch, G. S. (2003). The Family and the Internet: The Israeli case. Social Science Quarterly, 82, 329-339.

Ministerio de Tecnologías de la Información y las Comunicaciones. (2010). Percepción, usos y hábitos frente a las Tecnologías de la información y la Comunicación. Recuperado el día 12 de marzo del 2012, de: http://lacomunicacioncientifica.blogspot.com.es/2011/04/percepcion-usos-y-habitosfrente-las.html

Montero, L. y Leon,O.G. (2007). A guide for naming research studies in Psychology. (2007) International Journal of Clinical and Health Psychology, 7, 847-862.

Morrison, C. M. y Gore, H. (2010). The relationship between excessive Internet use and depression: A questionnaire-based study of 1,319 young people and adults. Psychopathology, 43, 121-126.

Muñoz-Rivas, M., J., Fernández, L. y Gámez-Guadiz, M. (2010). Analysis of the indicators of pathological Internet use in Spanish university students. The Spanish Journal of Psychology, 13, 697-70.

Muñiz, J. y Hambleton, R. K. (2000). Adaptación de los test de unas culturas a otras. Metodología de las Ciencias del Comportamiento, 2, 129-149.

Murdock, W. (2011). The relationship among family cohesion, gender, level of acculturation, and depression in Latino adolescents. United States: Proquest Umi Dissertation Publishing.

Observatorio Nacional de Telecomunicaciones y de la SI. (2011). Redes sociales en Internet. Recuperado el 1 de abril del 2012, de: http://www.ontsi.red.es/ontsi/es/estudios-informes/estudio-sobre-el-conocimiento-y-uso-de-las-redessociales-en-espa $\% \mathrm{C} 3 \% \mathrm{~B} 1$

Olson, D.H., Portner, J. y Lavee, Y. (1985). FACES III. St. Paul, MN: University of Minnesota. Ponce-Rosas, E.R., GómezClavelina, F.J., Terán Trillo, M., Irigoyen-Coria, A. y Landgrave- Ibáñez, S. (2002). Validez de constructo del cuestio- nario FACES III en español (México). Atención primaria, $30,624-630$.

Qualter, P., Brown, S.L., Munn, P. y Rotenberg, K. (2010). Childhood loneliness as a predictor of depression: an 8 year longitudinal study. European Journal of Child and Adolescent Psychiatry, 19, 493-501.

Rubio Gil, A. (2009). Adolescentes y jóvenes en red. Madrid: INJUVE.

Sánchez, A., y Martin, A.A. (2011). Informe Generación 2.0 2011. Hábitos de uso de las redes sociales en los adolescentes de España y América Latina. Recuperado el 24 abril del 2012, de: http://www.slideshare.net/ucjc/generacin-20-2011-hbitos-de-usos-de-las-redes-sociales-en-los-adolescentes-deespaa-y-amrica-latina

Seguic, J. (2010). Estado de la Internet - Colombia. Recuperado el 20 mayo del 2012, de: http://www.slideshare.net/wsicdelarosa/reporte-iab-comscore-Internet-encolombia?src=related_normal\&rel $=6061491$

Segura, S., Posada, S., Ospina, M., y Ospina, H. (2010). Estandarización del inventario CDI en niños y adolescentes entre 12 y 17 años de edad, del municipio de Sabaneta del Departamento de Antioquia - Colombia. International Journal of Psychological Research, 3(2), 63 -73.

Shapira, N. A., Goldsmith, T.D., Keck, P.E., Khosla, U.M. y Mc Elroy, S. M. (2000). Psychiatric features of individuals with problematic Internet use. Journal of Affective Disorders, 57, 267-272.

Spyridou, A. y Fernández-Parra, A. (2008). Adaptación de diversos instrumentos para la evaluación de la Soledad y Aislamiento Social en niños y adolescentes. Manuscrito no Publicado. Universidad de Granada, Granada, España.

Tabernero, C., Aranda, D. y Sánchez - Navarro, J. (2010). Juventud y tecnologías digitales: espacios de ocio, participación y aprendizaje. Revista de Estudios de Juventud, 88, 77-96.

Torres Galvis, Y. (2010). Situación de salud mental del adolescente. Estudio Nacional de Salud Mental Colombia. Bogotá: Ministerio de la Protección Social. Recuperado el 9 de marzo del 2012, de http://www.onsm.gov.co/

Unicef (2011). Estado mundial de la infancia, recuperado el 2 de abril del 2012, de: http://www.Unicef.org/devpro/files/ SOWC_2011_Main_Report_SP_02092011.pdf

Valencia, G. y Andrade - Palos, R. (2005). Validez del Youth Self Report para problemas de conducta en niños mexicanos. International Journal of Clinical and Health Psycho$\log , 5(3)$ 499-520.

Viñas, P. F. (2009). Uso autoinformado de Internet en adolescentes: perfil psicológico de un uso elevado de la red. International Journal of Psychology and Psychological Therapy, 9(1), 109-122. 